\title{
Construction of a Model for Discriminating between Electroencephalographic Patterns at the Time of Incorrect Inputs Based on Sensitivity Spectrum Analysis
}

\author{
Raita Ohori, Daiki Shinkai, Yoshimitsu Nagai, and Syohei Ishizu \\ Department of Science and Engineering \\ Aoyama Gakuin University, Japan \\ C5610167@aoyama.jp
}

\begin{abstract}
We consider the possibility of prior discovery of slip-type human error by quantifying the "distraction" state that precedes it. We investigated brain activity during the task of keyboard-based text input taking incorrect input as a slip-type error. We used sensitivity spectrum analysis to quantify four output states. We attempted to detect in correct input by discriminating between the output of the distraction state before and after incorrect input and that of the state before correct input.
\end{abstract}

Keywords: Sensitivity spectrum analysis, electroencephalogram, event-related potential, distraction, error recognition, human error.

\section{Introduction}

Recently, when buying and selling stock, a certain company accidentally ordered "one share at 610,000 yen" instead of the intended "610,000 shares at 1 yen," and thereby incurred a substantial financial loss. Such failures are referred to as human error. Early detection of human error is important in preventing such losses. It is difficult to prevent all human errors, but measures should be taken to limit them. Examples of measures intended to limit human error are records management and fool proofing. Records management allows human error to be tracked by recording and accumulating data, and can prevent the re-occurrence of an error by information sharing. Fool proofing entails assuming that any possible error will happen, and taking measures to avoid problems after an error occur. In either case, however, human error is not prevented from happening beforehand.

According to British cognitive psychologist J. Reason, there are two types of human error: planning failures and practice failures. The former is called a slip or a lapse, and the latter is called a mistake. In the case of a slip, the plan itself is correct, but there is a failure in its implementation. A lapse is an error in which the plan is forgotten during the course of implementation. In a mistake, implementation of the intended plan is correct, but the plan itself is flawed. Slips in particular are related to failures of attention, and these are the only errors in which action is routine and there is no consciousness of the correctness of the plan. In other words, there is a failure to pay sufficient attention, for instance, due to fatigue or worry, and the actor enters a 
"distraction" state that causes the error. We therefore wondered whether it might be possible to detect slips beforehand by quantifying the distraction state.

It is known that monitoring the activity of the human brain can reveal changes in mental state, such as entering a distraction state. Musha [1] proposed using electroencephalography to quantify the sensitivity of mental states such as "anger", "satisfaction", "sorrow", and "pleasure". This technique is called the sensitivity spectrum analysis method. Because mental states are complex, it is difficult to directly specify the relationship between active parts of the brain, even using information on the occurrence or non-occurrence of brain waves, such as alpha and beta waves. Sensitivity spectrum analysis uses three elements-time, frequency, and space - to quantify a sensitivity state by assembling a matrix that assumes feelings as an output vector. By replacing the four states of "anger", "satisfaction", "sorrow", and "pleasure" with states pertaining to the occurrence of error, we hoped that prior discovery of slip-type human error would be possible if we could assume a distraction state in the output.

In this study, we used keyboard-based text input into a PC as a task for investigating the distraction state. It is common for slips to occur when performing such a task. As performed by an expert, text input is largely automated: in cases of incorrect input, the plan for inputting is itself correct, but at some stage, a wrong key is typed and thus failure of implementation occurs. In other words, the incorrect input is slip-type human error. We consider a keyboard input task and use electroencephalography to quantify the distraction state before the incorrect input, which is a slip-type error. By quantifying the distraction state, we can expect prior discovery of slip-type human errors

The purpose of this study is quantification of the distraction state that precedes slip-type human errors. We used a PC-based text input task and considered incorrect input as a slip. We used sensitivity spectrum analysis to quantify the mental states. We converted electroencephalographic data into four output states of inputs and attempted to detect incorrect inputs by discriminating between the output of the distraction state before and after incorrect input and that of the state before and after correct input.

\section{Electroencephalography and Sensitivity Spectrum Analysis}

\subsection{Electroencephalography}

Electrical activity in the brain is measured as brain waves. Here, we describe frequencies and event-related potentials that are characteristic reactions, and their measurement values.

An electroencephalogram contains delta waves, theta waves, alpha waves, and beta waves, which are classified according to their frequency:

Delta waves $(0.5-4 \mathrm{~Hz})$

Theta waves $(4-8 \mathrm{~Hz})$

Alpha waves $(8-13 \mathrm{~Hz})$

Beta waves (13-40 Hz)
Sound sleep

Drowsiness

Mental rest

Mental activity 


\subsection{Event-related Potential (P300)}

A limitation of electroencephalographic analysis is that signals resulting from a given stimulation of a specific sense are weak. The addition average law is used to overcome for this. The addition average law is applied to electroencephalography by performing the same stimulation tens of times, recording the electroencephalogram each time, and matching recorded data from the times of stimulations to add and average the signals. Waves irrelevant to the target stimulation will have different measurements each time, and so eventually will be averaged out and become approximately flat. Confirmed changes in the electroencephalogram are called eventrelated potentials. Regardless of the type of stimulation, significant changes occurring 0.3 s before or after are called P300. It is said that the P3, Pz, and P4 spots of the international 10-20 system can confirm many P300 waveforms.

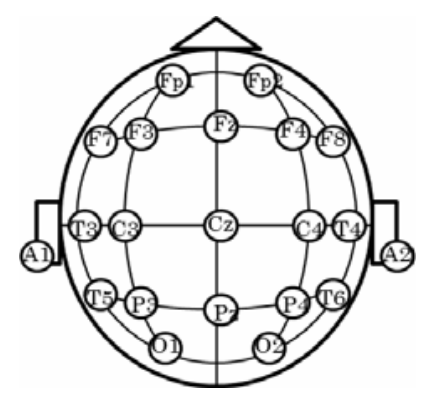

Fig. 1. Electroencephalogram measurement positions of the international 10-20 system

\subsection{Sensitivity Spectrum Analysis}

We shall next discuss the principles of sensitivity spectrum analysis. Ten electrodes are arranged on the scalp, and the electroencephalogram is recorded. The equation in Formula (1) is then used to find the cross-correlation coefficient between the electrodes. Cross-correlation coefficients have the following advantages:

1. They are not influenced by individual differences in magnitude between electroencephalogram amplitudes.

2. They can express phases between the parts of the electroencephalogram, such as polarity relations.

3. They can express both wave pattern information and information between parts at the same time.

$$
X_{i, j, k}=\frac{\sum\left(X_{i, k}(t) X_{j, k}(t)\right)}{\sqrt{\sum\left(X^{2}{ }_{i, k}(t)\right) \sum\left(X^{2}{ }_{j, k}(t)\right)}}
$$

In Formula (1), $X_{i, j, k}$ are the cross-correlation coefficients, $i, j=1 \sim 10$ are the measurement points, $k=\theta, \alpha, \beta$ are the three types of brain waves, and $t$ is time. 
For 10 chronologically ordered measurements, 45 cross-correlation coefficients are obtained and 135 variables are obtained when every frequency is needed. The 135 variables, arranged in order, are called a state vector. Four states of feelings are assigned values of 0 or 1 , and four variables to express those levels are called a sensitivity vector. Linear conversion is used to derive a sensitivity vector from the state vector and a $135 \times 4$ matrix is used for the conversion. This is called a sensitivity matrix (Fig. 2).

$$
\left(\begin{array}{l}
a_{1,1}, a_{1,2} \ldots a_{1,135} \\
a_{2,1}, a_{2,2} \ldots a_{2,135} \\
a_{3,1}, a_{3,2} \ldots a_{3,135} \\
a_{4,1}, a_{4,2} \ldots a_{4,135}
\end{array}\right) \times\left(\begin{array}{c}
x_{1} \\
x_{2} \\
\vdots \\
x_{135}
\end{array}\right)+\left(\begin{array}{c}
c_{1} \\
c_{2} \\
c_{3} \\
c_{4}
\end{array}\right)=\left(\begin{array}{c}
z_{1} \\
z_{2} \\
z_{3} \\
z_{4}
\end{array}\right)
$$

Fig. 2. The sensitivity spectrum analysis model

In this study, we use sensitivity spectrum analysis to quantify the output of the distraction state before incorrect input and to quantify the output of other states before correct input in a keyboard-based text input task.

\section{Creating the Incorrect Input Sensitivity Matrix}

\subsection{Electroencephalogram Measurement Experiment}

Experiment Preparations. We used a non-adhesive electrode helmet (BFL-EH15) to record the electroencephalogram. Nine electrode positions were used: Fp1, Fp2, F3, F4, P3, P4, O1, O2, and Pz, as per the locations shown in Fig. 1. We used the right earlobe as a standard electrode, and conducted the measurement using monopole induction.

Signals sent from the electrodes underwent A/D conversion, and a processor box recorded, digitized, and filtered the data. We transferred output data for storage and analysis on a computer through a SCSI bus interface. During the experiment, we displayed words for keyboard-based text input on a computer in a soundproofed room, and electroencephalograms were measured at the time of incorrect inputs. The screen on the computer used for text input was partitioned into two areas. The lower partition displayed the sentence to be typed, and the upper partition was used for text input. 


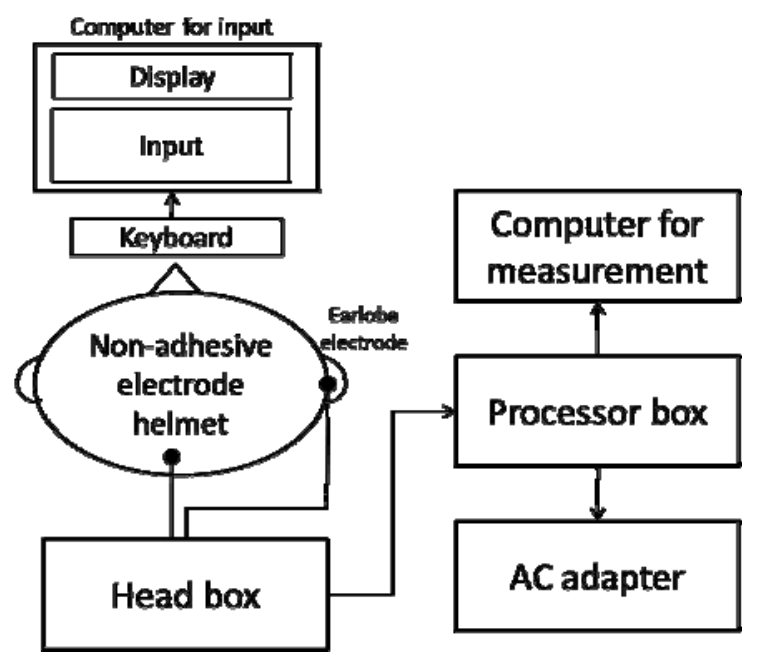

Fig. 3. Experiment overview

Experimental Procedure. The participants were nine healthy men aged 21-24 years old. We explained the text input measurement to them without mention of the incorrect input measurement, and after obtaining their consent, conducted the test according to the procedure given by steps $1-4$ below.

1. Participants sat in front of the text input computer and an electroencephalogram was recorded.

2. Participants closed their eyes and rested for $1 \mathrm{~min}$.

3. Each sentence consisting of around 100 kanji and hiragana characters was shown in the display partition of the computer screen.

4. Participants used the keyboard to input into the input partition the sentence displayed in the display partition.

We measured electroencephalograms for 5 types of sentences for each participant, with breaks of 1 min between inputting sentences.

Results of the Experiment. Table 1 shows the number of incorrect inputs from the nine participants (A-I).

Table 1. Number of incorrect inputs for each participant

\begin{tabular}{|c|r|r|r|r|r|r|r|r|r|r|}
\hline Participant & A & B & C & D & E & F & G & H & I & Total \\
\hline $\begin{array}{l}\text { Incorrect } \\
\text { inputs }\end{array}$ & 15 & 14 & 9 & 24 & 21 & 18 & 16 & 14 & 21 & 152 \\
\hline
\end{tabular}


Separately from data related to incorrect inputs, we also recorded data related to correct inputs. Funada has confirmed that P300 waveforms appear as a result of keyboard input processes [2]. In this knowledge of P300, we consider that a change can be seen in electroencephalograms approximately $0.3 \mathrm{~s}$ after an incorrect input. The time duration before occurrence of the waveform was taken to be $0.2 \mathrm{~s}$, because the average time before hitting the backspace after an error was $0.4 \mathrm{~s}$. We classified electroencephalographic recording before incorrect input as that related to the distraction state. We took the time duration of distraction to be $1.2 \mathrm{~s}$ (from $1.0 \mathrm{~s}$ before the incorrect input to $0.2 \mathrm{~s}$ after the incorrect input). We used the same time duration for the correct input state. We found cross-correlation coefficients between the electrodes to use in determination of the time duration. We found cross-correlation coefficients at the times of incorrect inputs and correct inputs in the distraction state and in the error recognition state during the time duration, and developed the sensitivity matrix under the assumption that we could detect in correct input from the relationship between the distraction state and the matrix value. In other words, if the discrimination precision of the matrix is high, the matrix value of the distraction state will rise before the incorrect input, and the matrix value of the error recognition state should rise after incorrect input. We show the time duration of the incorrect input in Fig. 4.

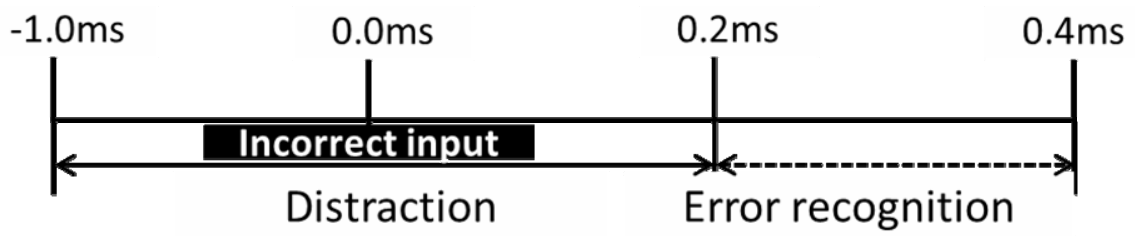

Fig. 4. The distraction state and the error recognition state in the time duration

\subsection{Creating the Incorrect Input Sensitivity Matrix}

Cross-correlation Coefficient. We recorded electroencephalograms showing theta waves, alpha waves, and beta waves from nine measurement points during each time duration. We found 108 cross-correlation coefficients to interlace with provided data using the equation shown in Formula(1).

Next, in the cross-correlation coefficient of the electroencephalographic data for the error recognition state and the normal input state, we show pairs of significant differences between averages on the horizontal axis (Fig. 5). In Fig. 5, we found that theta waves had many pairs of significant differences in the cross-correlation coefficient. In particular, the F3 measurement point showed the largest number of significant differences. Significant differences were also seen in Pz. We therefore had found a difference in the cross-correlation coefficient, and so considered the cross-correlation coefficient obtained when creating the sensitivity matrix to be useful. 


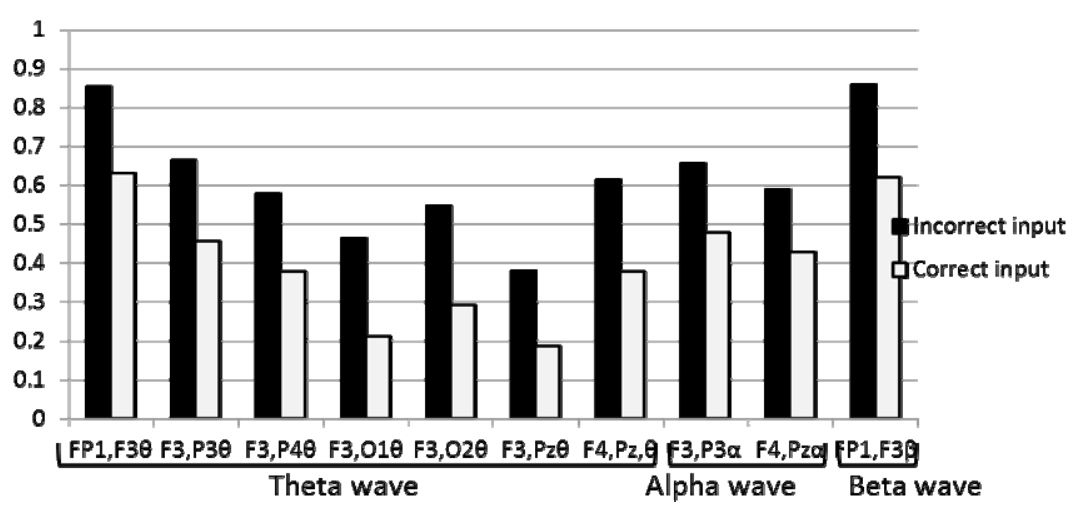

Fig. 5. The cross-correlation coefficient of electroencephalogram data of the incorrect input state and the correct input state

Creating the Incorrect Input Sensitivity Matrix. For the sensitivity matrix, we derived a matrix to output the state of error recognition and the state after normal input, and a matrix to output the state of distraction and the state before the normal input. We used the sensitivity spectrum analysis model of Fig. 2, creating a sensitivity spectrum analysis model that output data for times of incorrect input and correct input, since in this study we used the data from times of correct and incorrect input as substitutions for the four states of feelings (Fig. 6). The sensitivity vector before the time of the incorrect input outputs $Z_{1}, Z_{2}$. And, the sensitivity vector after the time of the incorrect input outputs $\mathrm{Z}_{1}{ }^{\prime}, \mathrm{Z}_{2}{ }^{\prime}$.

$$
\begin{aligned}
& \left(\begin{array}{l}
a_{1,1}, a_{1,2} \ldots a_{1,108} \\
a_{2,1}, a_{2,2} \ldots a_{2,108}
\end{array}\right) \times\left(\begin{array}{c}
x_{1} \\
x_{2} \\
\vdots \\
x_{108}
\end{array}\right)+\left(\begin{array}{c}
C_{1} \\
C_{2}
\end{array}\right)=\left(\begin{array}{c}
z_{1} \\
z_{2}
\end{array}\right) \\
& \left.\begin{array}{c}
\text { Sensitivity matrix of } \\
\text { Incorrect input }
\end{array}\right) \text { State vector Constant term Sensitivity vector }
\end{aligned}
$$

Fig. 6. Sensitivity spectrum analysis model at the time of the incorrect input

We assumed the cross-correlation coefficient that we found with the state vector. We used 1 as the sensitivity vector in each expression, and 0 for other values. We found the 108 coefficients by using multiple regression analysis in order to find the sensitivity matrix. We assumed the intercept found from multiple regression analysis as the constant term. In Table $2, a_{1}$ is the matrix of fixed number values before incorrect input (the distraction state), $a_{2}$ is the values before correct input, $a_{1}{ }^{\prime}$ is the values after incorrect input (the error recognition state), and $\mathrm{a}_{2}{ }^{\prime}$ is the values after the correct input. 
Table 2. Constant values of the incorrect input sensitivity matrix

\begin{tabular}{|l|l|l|l|l|l|}
\hline & 1 & 2 & $\ldots$ & 107 & 108 \\
\hline $\mathrm{a}_{1}$ & -1.9595 & 4.9017 & $\ldots$ & -7.5581 & 1.8951 \\
\hline $\mathrm{a}_{2}$ & 7.4453 & -76.8792 & $\ldots$ & 12.9451 & 11.0375 \\
\hline $\mathrm{a}_{1}$, & 2.2452 & 14.4860 & $\ldots$ & -2.9389 & -2.6061 \\
\hline $\mathrm{a}_{2}$, & 1.5692 & -2.3094 & $\ldots$ & 1.4934 & 1.3092 \\
\hline
\end{tabular}

Verification of Discrimination Precision. We verified the discrimination precision of the constructed matrix. We set $Z_{1}$ as the sensitivity vector value before an incorrect input (the distraction state), and $Z_{2}$ is the value before a correct input. Where $Z_{1}-$ $Z_{2}>0$, the state is before an incorrect input (the distraction state). Where $Z_{1}-Z_{2}<0$, the state is before a correct input. Likewise, we set $Z_{1}{ }^{\prime}$ as the sensitivity vector value after the incorrect input (the error recognition state), and $\mathrm{Z}_{2}{ }^{\prime}$ as the value after correct input. We had a participant input sentences and acquired 10,000 data points (we measured brain waves every $1 / 100 \mathrm{~s}$ ) in $100 \mathrm{~s}$. We detected incorrect input with these data during the time duration (0-120, 1-121...9880-10000) by a comparison with the matrix before the input. Likewise, we detected the incorrect input with these data during the time duration $(0-20,1-21 \ldots 9980-10000)$ by a comparison with the matrix after the input. We found the chronological order change of $Z_{1}-Z_{2}, Z_{1}{ }^{\prime}-Z_{2}{ }^{\prime}$ before and after the incorrect input, and we set the vertical axis as vector values, and the horizontal axis as time (Fig.7). Table 3 shows the discrimination precision results for all data.

Table 3. The discrimination precision of the sensitivity matrix for incorrect input

\begin{tabular}{|c|c|c|c|c|}
\hline & $\begin{array}{c}\text { Before } \\
\text { incorrect } \\
\text { input }\end{array}$ & $\begin{array}{c}\text { Before } \\
\text { correct } \\
\text { input }\end{array}$ & $\begin{array}{c}\text { After } \\
\text { incorrect } \\
\text { input }\end{array}$ & $\begin{array}{c}\text { After } \\
\text { correct } \\
\text { input }\end{array}$ \\
\hline Total data & 534 & 4890 & 534 & 4890 \\
\hline Discriminated data & 434 & 2542 & 427 & 3032 \\
\hline Discrimination rate & $88 \%$ & $52 \%$ & $83 \%$ & $62 \%$ \\
\hline
\end{tabular}

As shown in Fig. 7, the incorrect input is performed at $21 \mathrm{~s}$ (indicated by the double circle). The vector value of $\mathrm{Z}_{1}-\mathrm{Z}_{2}$ is large before the incorrect input (at 0.31 $\mathrm{s}$ ), and the vector value of $Z_{1}{ }^{\prime}-Z_{2}{ }^{\prime}$ becomes large after the incorrect input; thus, we could discriminate between states before and after incorrect input. Table 3 shows that, for all data, $88 \%$ and $83 \%$ of states respectively before and after incorrect input could be discriminated. However, the discrimination of states before and after correct input was $52 \%$ and $62 \%$, respectively. We attribute this discrepancy to the difficulty of treating data as equivalent despite influences such as differences between input words 
and differences in what is being thought at the time of the typing input. We were, however, able to quantify the state at the time of incorrect input using the sensitivity matrix.

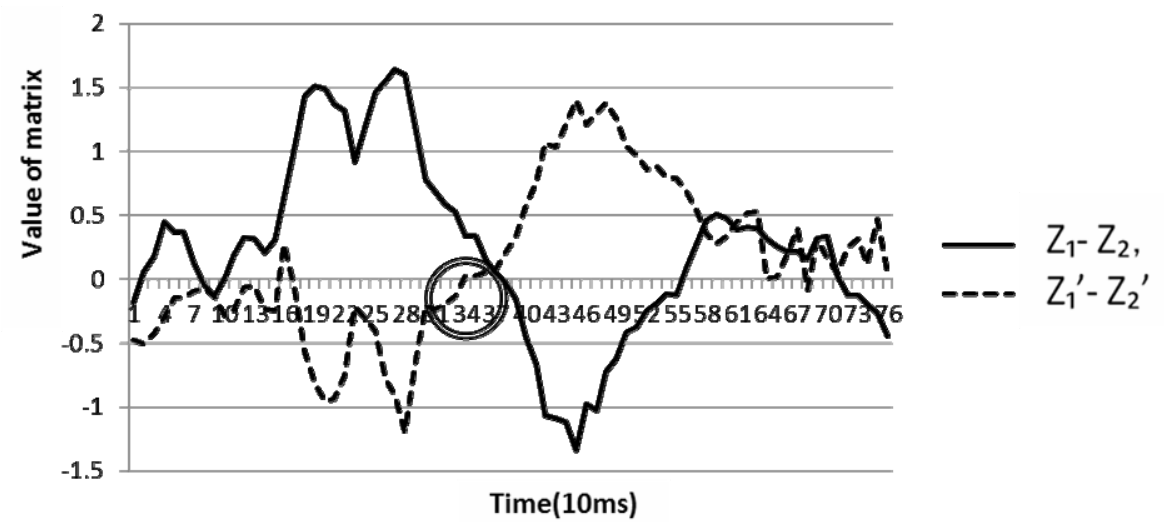

Fig. 7. The chronological order change of the sensitivity vector at the time of an incorrect input

\section{Conclusion}

In this study, we quantified the "distraction" state that precedes slip-type human error. We used a keyboard-based text input task and assumed incorrect input to be a slip. We examined four output states of inputs, using electroencephalography as a technique for quantifying the state, and using sensitivity spectrum analysis. We found that, with states of inputs output, it was possible to detect incorrect inputs by discriminating between the distraction state that precedes incorrect input and the state before correct input.

\section{References}

1. Araga, Y., Musha, T.: Numeric quantification of human emotions with EEGs. Bioscience \&Industry 59(10), 37-38 (2001)

2. Funada, M., Ninomiya, S.: A Discrimination Algorithm of Event Related Potentials Evoked by Characters. BioMed. (2004)

3. shimizu, T., Nakagawa, M.: Measurement of visual and sensational information by Images with Fractal Analyses of EEG. The Institute of Electronics, Information and Communication Engineers 108(219), 97-102 (2008) 